\title{
Qualidade de vida no trabalho: um estudo de caso das enfermeiras do Hospital Heliópolis
}

\author{
Life quality at work: a study of case \\ of the nu rses of Heliópolis Hospital
}

Lourdes Margareth Leite Pizzoli 1

1 Hospital Heliópolis, Divisão de Enfermagem. Rua Cônego Xavi er, 276, sala 8, térreo, 01231-030, São Paulo SP. megpizzoli@webcable.com.br
Abstract The arti cle pre sents a stu dy of case referring the verification of Life Quality at Work (LQW) of the nurses' population of Heliopolis Hospital, by means of indicators based on dimensions of the Richard Walton' model. These dimensions propound wide indicators which better adapt to the Brazilian socioeconomic culture, adapting to the community's organization structure where situ a tes the target population. The institution is public, characterized by high complexity specialties, with predominancein su rgical activity, considered reference hospital, located in São Paulo State, Brazil. The research made part of the dissertation approved for obtainment of the Master title in Sciences of the Administration and Human Values at the Capital Aca demic Center, in Novem ber 2002. Explo rato ry research, of field, qualitative in the fundamental elaboration of concepts for confection of the questionnaire, and quantitative regarding mensuration procedures of the closed answers, with statistical treatment through descriptive analysis and distribution of the frequencies of the variables. The re sults were positive regarding integration, social relevance, use opportunity and to the development of the capacities and negative regarding the absence recognition by work, absence project career, deficient communication and incompatible remuneration with the function.

Key words Life Quality at Work (LQW), Walton's Model, Nurses, Heliópolis Hospital
Resumo O artigo apresenta um estudo de caso referen teà Qualidade de Vida no Tra balho (QVT) da população de en fermei ras do Hospital Hel iópolis, medianteindicadores baseados nas dimensões do modelo de Rich ardWalton. Suas dimensões apresentam indicadores amplos que melhor se adaptam à cultu ra socioeconômica brasileira, adequando-se à condição organizacional da comunidade onde se situa a população-aho. A instituição é pública, caracterizada por especialidades de alta complexidade, predominância cirúrgica, hospital de referência, em São Paulo. A pesquisa fez parte da dissertação aprovada para mestrado em Gências da Administração e Valores Humanos no Centro Universitário Capital, em 2002. Pe squisa explo ratória, de campo, qualitativa na el a bo ração fundamental de concei tos pa ra confecção do questionário, e quantitativa nos procedimentos de mensuração das respostas fechadas, com tratamento estatísti co por meio de análise descritiva e distribuição das freqüências das variáveis abordadas. Os resultados apresentaram-se positivos quanto à integração, relevância social, opo rtunidade de uso e ao desenvolvimen to das capacidades; e negativos quanto à ausência de reconhecimento pelo trabalho, ausência de plano de carreira, co municação deficien te e remuneração incompatível com a função.

Palavras-ch ave Qualidade de Vida no Trabalho (QVT), Modelo de Walton, Enfermeiras; Hospital Heliópolis 


\section{Introdução}

Esperava-se que o Terceiro Milênio chegasse marcado por um domínio sereno das ciências, com precisão e con trole natural. O que se observou foi a ch egada de um século 21 caracteri$\mathrm{z}$ ado por eventos político-religiosos desafiadores, destrutivos, revelando que a humanidade ainda ignora os processos sociais, emoci onais e o intenso poder da força ideológica.

Numa era tecnológica que previa probabilidades, mediante planejamentos, con troles, índices e visualização do futu ro, refletiu a imprevisibilidade da gl orificação da máquina. Acreditando no poder da racionalidade e do intelecto, a evolução mostrou que esses eventos, mesmo associados a um progresso financei ro e educativo, não traduziram uma melhora da consciência social.

Sabemos mais das máquinas e dos mercados do que das motivações que nos fizeram inventar e institucionalizar essas coisas. Somos também ignorantes das emoções que nos levam a ter respostas exclusivas para todos os problemas sociais, que garante que esses males têm causas únicas. E que, conseqüen temente, qu em sabe disso tem o dever e, mais que isso, o di rei to de tomar o poder a qualqu erpreço para implementar uma nova e inusitada era de felicidade. Hitler, Stalin e Bin Laden são desse time. Bem como os velhos deterministas que lhes deram (e têm dado) farta munição ideológica (Damatta, 2002).

Explorando e de envolvendo mais o conc reto extern o, observamos que o interno humano se ex pressou sobrem a n ei ra na véspera do século 21, num mun do que se deparou com uma busca incessante por novos modelos tecnológi cos e administra tivos, no intui to de permi tir a gestão de mel h ores serviços, mediante o estudo para aprimoramento e compreensão das relações humanas.

Na edificação social e no intercâmbio cultural, veiculados cada vez mais intensamen te nos meios de comunicação, verificam-se extremos de situações, do fanatismo rel i gioso à desmedida ambição, com even tuais ch oques de crenças e valores, representan do o quan to desconhecemos do com portamento humano na construção de ambien tes e relações harmônicas e respeitosas, medianteas diferenças naturais.

Essas circunstâncias se refletem de forma in tensa nos ambien tes sociais, com destaque nas relações de trabalho. Para atualizar os últimos con cei tos de tecnologia e qualidade, con- sideramos imprescindível associar o com prometimen to dos funcion á rios dos mais variados níveis hierárqu i cos de uma em presa. Isso implica verificar o grau de satisfação e de participação e os impactos daí decorren tes e sua repercussão na eficácia or ganizacional.

Esses aspectos podem ser compreen didoscomo uma forma de rever o posicionamento das pessoas em relação ao trabalho e à comunidade. $\mathrm{O}$ estu do de indicadores para mensurar esses el em en tos su r giu com o desenvolvimen to do tema den omin ado Qual id adede Vida no Trabalho (QVT), na busca de novas formas de gerenciar o trabalho e inves tir no po tencial humano.

A verificação de índices de QVT pode trazer informações de fatores que interferem diretamente na satisfação e motivação pessoal e coletiva, com refl exos na excelência da estrutu ra e do serviço. Um estudo sobre esses elementos permite conhecer como as pessoas se sentem em relação a vários aspectos (tanto internos como ex ternos) da em presa e, a partir daí, gerenciar esses dados, tran sforman do essas inform ações em bases para a construção de estratégias que prom ovam o aumen to do envo lvim en to.

As em presas necessitam de pessoas motivadas e comprom etidas com seus objetivos e sua filosofia. Desse modo, o levantamen to das percepções dos funcionários em relação aos fatores intervenien tes da qualidade de vida no trabalho torna-se fundamental.

O trabalho pode ser visto como ação humana desenvo lvida num con texto social, que recebe influências de várias fontes, resultando nu ma ação recíproca en tre o tra b al hador e os meios de produ ção, que foi gradativamente limitado por condições socialmen te estabelecidas, ten do o ser humano como produto e produtor da soci ied ade na qual se insere. No percurso evolutivo das Ciências da Administração ob s erva-se acen tu ação do enfoque nas relações humanas como base para a motivação no trabalho.

Consideran do a motivação referência a um estado interno resultante de uma necessidade, a borda-se sua inferência na ex pressão do comportamen to. A identificação do motivo pode auxiliar na compreensão do comportamen to humano, mediante interpretações quanto às diferenças pessoais nas tendências motivacionais básicas, com reflexos na vida pessoal e, conseqüentemente, na vida de relação do trabalho. Mediante a cultura, a ex periência familiar e individual, c ada pessoa desenvolve forças de motivação que influ enciam no seu trabalho, con forme seus valores. 
As instituições hospitalares, com vistas à melhoria da própria qualidade de vida da população, também se en con tram nessa busca de modelos que favoreçam a qualidade na prestação de assistência.

Uma comunidade hospitalar, de modo geral, pode ser descrita como qualquer outra organização em presarial, em termos de gestão administra tiva, com resultados que podem sofrer influência tanto de fatores internos quan to externos à organização (Marquis \& Huston, 1999). Porém, pos sui características bastante peculiares, que envolvem atuações profissionais diret a m en te ligadas aos princípios de manutenção e recuperação da saúde, e, por con s eguinte, dos mananciais que sustentam a própria existência.

Desse modo, a equipe operacional de saúde, mais precisamente a profissional enferm eira, geralmen te é submetida às situações geradas pelas atividades ineren tes à função, envolvendo inúmeros elementos nega tivos proporcionados pelo ambien te caracterizado pela enfermid ade.

Pela ampla representividade do gênero feminino no exercício da profissão, preferimos o termo enferm eira para referência no decorrer do es tu do, não som en te pelo qu a n tit a tivo $(94,78 \%$ no Estado de São Paulo, segundo o Conselho Regi onal de Enfermagem do Estado de São Paulo - Coren/SP - em feverei ro de 2002 e 96,88\% no Hospital Heliópolis em julho de 2002), mas principalmen te pela característica profissional, cujos aspectos voltados ao cuidar muito se relaci onam ao perfil atribuído ao feminino, associado com princípios da maternidade, da amamentação e outras peculiaridades do gerar, cuidar e prover.

Levando-se em conta que os profissionais passam muitas horas dentro do ambiente de trabalho, se estas puderem ser agradáveis, as pessoas vão se sentir mais motivadas e, conseqüentemente, mais envolvidas com os objetivos da em presa (Gil, 2000). Con si deramos que esse envolvimento é fundamental, para o aumen to não só da produção, mas, principalmente, da quali d adedo trabalho e do aprimoram ento profissional.

O pres en te estu do se justifica por suas contri buições para a discussão teórico-em pírica da noção de Qualidade de Vida no Trabalho, fornecendo subsídios para o crescimento na adm inistração quan to ao Serviço de Enfermagem. Stacciarini (2000) observa que poucas foram as investigações sobre estresse ocupacional e qua- lidade de vida do enfermei ro na literatura nacional, que apenas sugerem que o trabalho da en ferm a gem é estressante, sem maior aprof u ndam en tonos aspectos que o caracterizam.

Os estudos sobre QVT ainda são rela tivamen te escassos no Brasil, principalmen te na área de enfermagem. Caracterizada em restabelecer o bem-estar alheio, a profissão de enfermagem comumente é subm etida a diversos fatores que afetam a sua qualidade de vida. Tal influência pode ter origem em fatores intrínsecos à natu reza de sua atividade laborial, mas também nas condições de trabalho geradas pela organização, o que pode influ enciar outros aspectos indivi duais da vida pessoal do prof issional, podendo, portan to, com prom eter a motivação.

Gilberto Linhares (2002), presiden te do Conselho Federal de Enferm a gem (Cofen), com enta que essa realidade é ainda mais chocante nos hospitais, on de profissi onais que procuram auxiliar na cura "vivem o paradoxo de estar tão ou mais su j ei tos às en ferm id ades do que o pacien te entregue aos seus cuidado s". Riscos laboriais como aciden tes biológicos (radiações, inadequações de equipamen tos e instrumentais) e deficiências de infra-estrutura como causa freq ü en te de lesões por esforço repetitivo (LER) e necessidade de duplo emprego para subsistência mais digna, são complicadores dos impactos desses profissionais que acompan ham o paciente e suas famílias durante as 24 horas do dia, mesmo quando estão enfrentando algum problema pessoal ou familiar. Não é por acaso que esse con ju n to de fatores produz nesse meio, em grande escala, probl emas psicológicos e - já se comprovou - envelhecimen to precoce.

Por afetar a socied ade inteira, a grave realidade vivida pelos profissionais de saúde está a reclamar um damor de toda a nação pela melhoria das condições de trabalho e dos salários da operosa e sacrificada categoria da saúde, em especial a en fermagem.

De forma geral, ob serva-se na estrutura organizacional da comunidade hospitalar a implantação do poderio médico com a apropriação do crescimen to de saúde e o gerenciamento do processo de trabalho em saúde. Essa circunstância é decorrente da identificação dos médicos com as classes dominantes nos diversos momen tos históricos, produzindo discurso, saber e tecnologia necessários à manutenção do st a tus quo $e$ a ordem social estabel ecida, di ferentem en te do senso comum, que en tende que a su- 
premacia médica no setor se deve à superioridade do saber médico (Pires, 1989).

Essa supremacia do conhecimento começou a se fragmentar, acen tu a n do-se com o su rgim en to e crescimen tovertiginoso das especialidades médicas, gerando uma perda da visão total do humano, não somente do enfermo, mas até nas relações da comunidade hospitalar (Weil, 2000).

Mi n ayo (1999) con si dera que a relação positivista na prática médica se manifesta em três expressões básicas:

- Na concepção do binômio saúde-doença apenas como fenômeno biológi co individual, em que o aspecto social entra, porém, compreen dido como modo de vida e apenas como variável, ou é desconhecido e omitido;

- Na valorização tecnológica excessiva e na capacidade absoluta da Medicina de erradicar as doenças;

- Na dominação corpora tiva dos médicos em relação aos outros campos do conhecimento, adotando-os de forma pragmática (a sociologia e a antropologia consideradas importantes apenas para fazer questionários, produzir informes culturais, ensinar alguns con cei tos bási$\cos )$; no tratamen to subalterno dado aos outros profissionais da área (enfermeiros, assisten tes sociais, nutricionistas, atendentes); em relação ao senso comum da população, numa tentativa nunca totalmen te vitoriosa, de desqualificá-la e absorvê-lo.

Observando como base hier á rquica a autoridade médica, principalmente dentro da comunidade hospitalar, para os indivíduos que atuam indiretamente na assistência ou com funções de men or evidência nas condutas terapêuticas ou diagnósticas, pode-se ter a ilusão de men or grau de importância. Além de el evado índice de estímulos estressores, $\mathrm{n}$ a tu ral num ambien te on de predomina a enfermidade, é freqü en temente com entada a insatisfação pela ausência de reconhecimen to das atividades profissionais.

Considerando a importância desses elemen tos na área de saúde, principalmente numa profissão de atuação essencial, de senvolveu-se es te trabalho com a finalidade de estu do do tema Qualidade de Vida no Trabalho (QVT), baseado no Modelo de Richard E. Walton, na comunidade hospitalar de órgão públ i co, através de um estu do de caso das enferm eiras do Ho spital Heliópolis.

\section{Objetivos}

Tal pesquisa intentou obter subsídios que elucidassema situação profissional e forn ecer dado s p a ra con tribuir na ree s trutu ração organizacional para melhoria da qualidade de vida no trabalho. Limitou-se aos profissionais de nível superi or, pois de senvo lvem ativi d ades de assistência con comitan tes ao geren cia m en to operacional da equipe de enfermagem sob seus cuidados que, particularmente na Insti tuição objeto do estudo, necessitam gerenciar amplas equipes de trabalho, além das atividades administrativas e de intercâmbio multi profissional e de mediações com familiares e órgãos institucionais.

Consideran do as situações ineren tes à função da en ferm ei rano âmbi to hospitalar, envo lven do os elemen tos que rodeiam os procedimentos mediante as enfermidades e a própria e s trutura organizacional, bu s cou-se identificar os fatores que pudess em com prom eter a motivação, o des envolvimento e a atuação profissional.

\section{Metodologia}

Esta pesquisa pretendeu iden tificar os fatores que possam esti mulara des mo tivação, atuantes nas enferm eiras do Hospital Heliópolis, verificando as percepções das mesmas diante dos fatores de QVT. Essa relação entre QVT e produtividade é adaptada do modelo de Richard E. Walton. Desenvo lveu-se como instrumen to de coleta de dados um questionário para possibilitar a avaliação do nível de satisfação das enfermeiras, verificandoos vários fatores que interferem no cotidiano e, a partir da percepção manifestada, mostrar a importância da avaliação da Qualidade de Vida no Trabalho (QVT).

Estabel ecemos alguns con cei tos sobre motivação para operacionalizar a mensuração, a través de aspectos de satisfação e insatisfação, na busca de envo lvimen to e sen ti do para o exec utor. Abordamos também fatores de comunicação que possam interferir nas relações e estimular ou prejudicar as forças produtivas e elementos que podem interferir na quali d ade e motivação den tro da área da saúde no serviço públ i co.

A pesquisa se constituiu em estu do exploratório e de campo, de caráter qualitativo na el a boração fundamental de con cei tos para confecção do questionário, e quantitativo quan to aos procedimen tos de mensu ração das respostas fechadas. As perguntas abertas intentaram 
obter manifestações espontâneas, suscitadas pelo próprio questionário, para verificação de su rgim en tode outros fatores não incluídos.

O tratamento final constituiu-se na construção de tabelas de freqüência e percentuais qu a $\mathrm{n}$ to aos fatores pertin en tes a cada dimensão considerada, den tro do modelo de Walton.

A partir dos dados levantados, procedeu-se ao tratamen to estatísti co por meio de análise descritiva e distri buição das freqüências das variá veis abordad as. Com esse tratamento, verificaram-se as diferenças significativas de comportamen to pela estatística de freqüências cruzadas, ch egan do-se aos result ados finais.

Dentre os 58 profissionais ativos, obtivemos 54 devoluções de questionários, correspon den do a $93,10 \%$ res pondentes.

O levantamen to de dados para a presen te pesquisa foi efetuado por questionário como instrumen to de investigação, aplicado duran te o perí odo de julho e ago s to de 2002, após aprovação do proj eto pelo Comitê de Ética em Pesquisa do Complexo Hospitalar Heliópolis e posterior con cordância de participação pelas pessoas, mediante o preenchimento do termo de Consentimento Livre e Escl a recido.

$\mathrm{Na}$ hipótese inicial, con si derou-se que o nível de satisfação das enfermeiras do Hospital Heliópolis poderia ser verificado em questionário baseado no modelo de QVT de Walton. Tal hipótese foi proposta baseada na abordagem dos aspectos elementares à realização e situação no ambi en te de trabalho, enfocando simultaneamen tefatores higiênicose motivacionais, os quais constituem os 21 fatores pertinen tes às oi to dimensões con si deradas no Modelo de Walton, facilitandoa verificação de deficiências percebidas que possam afetar diretamen te a QVT.

A pesquisa qualitativa não possui como pre s su po s to uma gen eralização de seus resultados e, portan to, es tes são válidos som en te para o hospital em estudo, den tro do con tex to espaço-temporal e limites de qualquer outra natureza em que o trabalho foi desenvolvido e os dados coletados. Esses limites são determinados pela dinâmica que caracteriza a organização como fen ô m eno social que repre s en $t a$.

\section{Apresentação dos resultados}

O levantamen todas va riá veis dem ográficas dem on s trou uma população basicamen te con s tituída pelo gênero feminino, apresentando um acúmulo de atividades assistenciais e gerenciais, caracterizado por acentuado deficit no quantita tivo de profissionais, com repercussão em praticamen te todos os aspectos avaliados. Verificou-se que cerca de $72 \%$ das respon dentes referi ram que a escolha da profissão foi vinculada à viabilidade de auxilio ao próximo necessitado, carente ou enfermo, e ex pressões de realização e espíritohumanitário.

\section{Síntese dos resultados obtidos em todas as dimensões e seus respectivos fatores}

1 - Com pensação justa e adequ ada (-): Predominância maciça de insatisfação.

a - Renda adequ ada (-): Apresen tou-se como um fator nega tivo, não sendocompatível com a responsabilidade e a importância da atividade.

b - Eqüidade interna (-): Não há muita diferença en tre tempo de trabalho e experiência, com result ado negativo.

c - Eqüidade ex terna (-): Apre s en tou-se como um fator negativo, em que o salário pago não é o prati c adono merc ado.

2 - Condição de trabalho (-): Predominância maciça de insatisfação.

a - Jornada de trabalho (-): Insatisfação quan to ao estresse pela carga mental e desgaste não somen te rel acionado à própria característica da profissão, mas, principalmente, pelo nú$m$ ero de en ferm eiras mu i to aquém do nece s sário, bem como quantitativo geral de profissionais de en ferm a gem redu zi do.

b - Ambiente físico seguro e saudável (-): Condições físico-ambi entais prejudiciais à saúde, reforçadas com sobrecarga na distribuição de tarefas. Também negativo quan to a materiais e equipamentos, in ovações tecnológicas e Equipamentos de Proteção In divi dual (EPI).

3 - Oportunidade de uso e desenvolvimento de capacidades (+): Aspectos equilibrados, com con cen tração em satisfação.

a - Autonomia (+): Possuem autonomia para decisão e conhecimento suficiente para de senvo lver suas tarefas.

b - Significado da tarefa (+): Percebem como de sua res ponsabilidade o resultado alcançado.

c - Identidade da tarefa (+): Consideram importantes as ativid ades desenvo lvidas, bem como reconhecem a importância para que o hospital de senvo lva seus obj etivos. 
$\mathrm{d}$ - Varied ade da habilid ade (+/-): Utilizam plenamente seus conhecimentos, porém a Insti tuição po u co inve s teem capacitações.

e - Retroi n formação (+): Avaliam em parte os resultados finais de suas atividades, conhecendo parcialmente a estrutura completa de funcionamento do hospital, e consideram pos i tivo o apoio de seus su peri ores imedia tos.

4 - Oportunidade de crescimen to e segurança (+/-): As pectos distribuídos entre satisfação e insatisfação de forma eqüitativa.

a - Possibilidade de carreira (+/-): Não possu em plano de cargos e carreira definido e percebem que a Insti tuição não va l oriza e reco$\mathrm{n}$ h ece seu trabalho.

b - Crescimen to profissional (+/-): A Instituição não possui sistema de incen tivo à en fermagem para prosseguir seus estudos formais, porém oferece condições internas de crescimen to pela prática.

c-Seg u rança de em prego (+): Possu emsegurança e estabilidade no em prego, por tratarse de vínculo públ i co, efetuado por con curso.

5 - Integração social no trabalho (+): Aspectos equilibrados, com con cen tração em satisfação.

a - Igualdade de oportunidade (+/-): A discriminação funcional foi a que revelou índices significativos.

b-Relacion a men to (+): Re sult adopo si tivo com relacionamen tos considerados satisfatórios. Também em relação ao senso comunitário e liderança e participação da gerência, com col a boração um po u co com prom etida.

6 - Constituciomlismo (+/-): As pectos distribuídos en tre satisfação e insatisfação de forma eqüitativa.

a - Respeito às leis e direitos trabalhistas (+/-): Não são efetuados exames médicos. As leis trabalhistas são cumpridas satisfatoriamente.

b - Privacid ade pessoal (+): Resultado po$s$ i tivo no res pei to pessoal.

c - Liberdade de expressão (+): Predominância de satisfação.

$\mathrm{d}$ - Normas e rotinas (+/-): Não possuem acesso para deb a te de normas gerais. As rotinas internas são mais abertas.

7 - Trabalho e espaço total da vida (+/-): Aspectos distribuídos en tre satisfação e insatisfação de forma eqüitativa.

a - Pa pel balance ado do trabalho (+/-): Pouca repercussão das ocorrências no lar, com equ i- líbrio entre trabalho e lazer. Não de s envo lvem a tivid ade física.

8 - Relevância social da vida no trabalho (+): As pectos equilibrados, com concentração em satisfação.

a - Imagem da empresa (+): Sa tisfação quanto ao orgulho por trabalhar na Instituição, por s eu prestígio, imagem, e pela auto-realização no trabalho.

O modelo analíti co adaptou-se adequadamente com êxito na verificação de aspectos el ementares à realização e situação no ambiente de trabalho, enfocan do simultaneamen te fatores higiênicos e motivacionais.

Verificamos que há equilíbrio entre as dimensões, poden do situar-se a Qualidade de Vida Total das en fermei ras do Hospital Heliópolis como razoável em alguns aspectos e bastante comprom etida em outros. Elevada con centração em satisfação nas dimensões que abrangem aspectos sociais, de relacionamen to e desenvo lvim en todo potencial. Em con trapartida, há insatisfação resultante nos aspectos que a bordam a valorização, compensação, condições de trabalho e investimen to na educação formal do profissional.

\section{Considerações finais}

Dor, doença e morte fazem parte do cotidiano e somam-se à angústia e à ansied ade do pac i ente com a integrid ade física com prom etida, com famílias transtornadas, intermediados com a realização de procedimentos assistenciais desconfortáveis, dolorosos, invasivos, num ambiente estranho, desnudando-os em seu desequilíbrio, ex pondo-os em sua fragilidade.

Além disso, fa tores constituintes da própria estrutura organizacional podem com prometer diretamente o de s envo lvim en toe a atuação, como ausência de recon hecim en to pelo trabalho, falta de plano de carreira, comunicação deficiente, falta de planejamento, salário incompatível com a função ou muitoabaixo do mercado. Essa associação pode colocar em risco a motivação e a satisfação, vindo a contribuir, conseqüen temente, para uma baixa produtivi$\mathrm{d}$ ade e qu eda na qualidade do serviço pre s t ado.

A abord a gem de aspectos que afetam o indivíduo e o grupo, principalmen te em uma comunidade de saúde de serviço públ i co, c a racteriza a importância dessa pesquisa, poden do 
propiciar a criação de estratégias para reestrutu ração que favoreça a melhoria das condições de trabalho, com repercussão no aumen to de produtividade e qualidade de assistência. Ainda são caracterizadas as peculiaridades voltadas às diferenças de gênero e suas relações com índices de estresse.

Os resultados apontam aspectos positivos envo lven do principalmen te a integração e a relevância social, bem como a oportunidade de uso e des envo lvimento das capacidades; porém a pontam aspectos negativos, como a ausência de reconhecimento pelo trabalho, falta de plano de carrei ra, comunicação deficiente e remu$\mathrm{n}$ eração incompatívd com a função. As re s pondentes expressaram um índice final discretamen te maior na graduação de satisfação.

O papel funcional e social da enfermeira, na cultu ra organizacional de uma comunidade hospitalar, pode gerar conflitos em maior ou menor grau com suas características individuais, o que explica as variações expressas nas respostas, comprometendo a motivação, e podendo gerar sofrimen to físico e psíquico, que, somados a outros fatores, são causadores de estresse.

Observa-se que a profissão ainda carrega muito dessa concepção de sacrifício e autoabandono, de s con si deran do as pró prias nece ssidades básicas, com pouco questionamento dos próprios limites físicos, emocionais, pessoais, em nome da manutenção do papel competente na atividade e função, mesmo que em detrimento pessoal. Permanece como pano de fundo um arquétipo de heroísmo, ressaltandose um trabalho que não visa à recom pensa financeira.

O orgulho do trabalho em si e na Instituição se conflitua com uma remun eração considerada injusta e condições de trabalho insatisfatórias pela sobrecarga de tarefas numa profissão já por si estressante. A enfermagem possui percen tual predomin a n te de mulheres, corroborado pela presen te pes quisa e pelos dados das en tid ades oficiais de estatística que, ao longo da história, foram bastante refreadas em suas expressões, e ainda hoje refletem dificuldades no auto-reconhecimen to, no auto-respei to e no autoc uid ado.

Consideramos que a produtividade em en ferma gem não possa ser medida por quantidade de procedimen tos e economia de materiais, pois os resultados de sua atuação nem sempre serão visíveis, palpáveis ou mensuráveis. $\mathrm{O}$ cuidado em enferm a gem nos parece di- retamente ligado ao envolvimen to e ao comprometimento da pessoa com a Insti tuição e com a profissão.

Para que um trabalho tenha importância, não é necessário envolver assun tos de grande significad históri co; en tretan to o trabalho precisa de um sentido para quem o faz. A pessoa deve sentir que é um trabalho que vale a pena $\mathrm{s}$ erfei to, que reforça sua auto-imagem e faz com que ela se sinta parte de um processo maior.

Com base nesses dados, ob s erva-se que mu itas intervenções podem ser efetuadas, a tenu a ndo os índices de insatisfação, favorecendo a motivação e prom oven do condições para melhorqualidade na assistência.

Considerando a atuação de enfermagem, no âmbi to hospitalar, envolvida em um trabalho de gru po multi profissional, acredita-se que, para se efetuar uma abord a gem de terapia mu ltidimensional, numa con cepção holística, é necessário que os membros da equipe de saúde continuem se especializando nos vários campos, porém compartilhando essa con cepção e efetuan do uma estrutura con ceptual comum, para uma comunicação eficien te e uma sistematização dessa integração.

É possível criar estratégias para lidar com algumas incoerências do dia-a-dia: se para com o ser mais próximo de nós, que somos nós mesmos, não con seguimos perceber nossa importância, i dentificar nossas necessidades, res erva r-nos um tratamen to carinhoso e paciente, como é que conseguiremos efetuar esses valores com auten ticid ade para com o outro?

O mesmo se aplica à Instituição. Se para com os que constituem seu próprio "corpo" de trabalho, seja o corpo clínico, o de enfermagem, ou qualquer outro componente da área da equipe de saúde, ou ainda para os que lhe dão suporte, não há investimento, atenção, nem busca na identificação de obstáculos à harmonia e equilíbrio nas relações de trabalho, o que será projet ado realmen te na assistência? Não aten den do às necessidades de seu próprio corpo funcional, propicia condições para a proliferação de elementos patogênicos, que podem levar a or ganização a estados en fermos e, até mesmo, à própria morte, pela falência de s eus múltiplos “órgãos”.

Não pode existir uma plena qualidade organizacional concomitante ao detrimento da qualidade de vida de seus funcionários. Desse modo, con sideramos que verificar as insatisfações pode forn ecer informações significativas para a construção de estratégias vol t adas ao de- 
senvo lvimento de reformas organizacionais. Só assim, é possíved gerar condições para o crescimen to profissional pessoal e da comunidade, que poderá ser ex presso numa assistência mais produtiva, segura e de qualidade inquestionável, com garantia na própria saúde da organização.

\section{Referências bibliográficas}

Almeida MCP \& Rocha JSY 1989. O saber de en fermagem e sua dimensão prática. Cortez, São Paulo.

Conselho Regional de Enfermagem do Estado de São Paulo 2002 (COREN/SP). Ofício 0789/2002/PRSG PRCI-30022. E-mail em 19 fev. 2002 enviado pela Dra. Ruth Miranda deCamargo Lei fert, COREN-SP1.104, Presidente do COREN-SP.

Damatta R 2002. O que há de novo no ano velho? O Estado de S. Paulo, 3 nov. 2002. Cad 2 (D8).

Fernandes EC 1997. Qualidade de vida no tra balho: como medir pa ramel ho rar. Casa da Qualidade, Sa lvador.

Gil AC 2000. Administração de recu rsos humanos: um enfoque profissional. Atlas, São Paulo.

Linhares G 2002. Saúde da enfermagem. Disponível em $<$ http://www. portalcofen.com.br/secoes/section_int. asp? InfoID $=515 \&$ EditionSectionID $=18 \&$ SectionPa rentID=\&id_lingua $=>$ Ace $s$ s ado em 18 out. 2002.

Ma rquis B \& Huston CJ 1999. Administração e liderança em enfermagem: teoria e aplicação. Artmed, Porto Al egre.

Mi n ayo MCS 1999. O desafio do conhecimen to: pesquisa qualitativa em saúde. Hucitec, São Paulo.

Pires D 1989. Hegemonia médica na saúde e a enfermagem: Brasil 1500 a 1930. Cortez, São Paulo.

Staccia rini JMR 2000. E s tresse ocupacional, satisfação no trabalho e mal-estar físico e psicológi co em enfermeiros. Nursing 20(3):30-34.

Weil P 2000. A mudança de sentido e o sentido da mudança. Record: Rosa dos Tempos, Rio de Janei ro.

Arti go apre s en t ado em 4/11/2004

Aprovado em 27/7/2005

Versão final apre s en t ada em 27/7/2005 